\title{
Generic "Unnecessary" Quantum Critical Points with Minimal Degrees of Freedom
}

\author{
Chao-Ming $\operatorname{Jian}^{1}$ and Cenke $\mathrm{Xu}^{2}$ \\ ${ }^{1}$ Kavli Institute of Theoretical Physics, Santa Barbara, CA 93106, USA \\ ${ }^{2}$ Department of Physics, University of California, Santa Barbara, CA 93106, USA
}

\begin{abstract}
We explore generic "unnecessary" quantum critical points with minimal degrees of freedom. These quantum critical points can be avoided with strong enough symmetry-allowed deformations of the Hamiltonian, but these deformations are irrelevant perturbations below certain threshold at the quantum critical point. These quantum critical points are hence unnecessary, but also unfine-tuned (generic). The previously known examples of such generic unnecessary quantum critical points involve at least eight Dirac fermions in both two and three spatial dimensions. In this work we seek for examples of generic unnecessary quantum critical points with minimal degrees of freedom. In particular, in three dimensional space, we identify two examples of such generic unnecessary quantum critical points. The first example occurs in a $3 d$ interacting topological insulator, and it is described by two $(3+1) d$ massless Dirac fermions in the infrared limit; the second example occurs in a $3 d$ topological superconductor, and it is formally described only one $(3+1) d$ massless Dirac fermion.
\end{abstract}

PACS numbers:

\section{INTRODUCTION}

A quantum critical point (QCP) is usually found between two different phases of matter with qualitatively different properties. A generic QCP has only one symmetry allowed relevant perturbation, which is the tuning parameter of the quantum phase transition, in other words a generic QCP is "unfine-tuned". In fact, the existence of a generic QCP in a phase diagram usually implies that the two phases on the opposite sides of the QCP are both stable fixed points under renormalization group (RG), and the QCP is unavoidable (or "necessary"), meaning there does not exist a smooth adiabatic route in the phase diagram that connects the two sides of the QCP, no matter how the Hamiltonian is deformed as long as certain symmetry is preserved. The simplest example that illustrates this common wisdom is the transition of the quantum Ising model in any spatial dimension. This Ising QCP is sandwiched between a disordered phase which preserves all the symmetries, and an ordered phase with spontaneous Ising $\left(Z_{2}\right)$ symmetry breaking. The Ising QCP is "necessary", meaning if one tries avoiding this QCP by deforming the Hamiltonian, the best one can do is to drive the QCP into a first order transition across a tricritical point, i.e. there is no smooth route that connects the disordered and ordered phases.

The Ising QCP is a quantum analogue of the classical Ising transition, and it is sandwiched between two phases with classical analogues at finite temperature. The study of quantum many-body systems have revealed that the quantum phases are far richer than classical phases, examples include the topological phases ${ }^{1}$ and symmetry protected topological phases ${ }^{213}$ (such as topological insulators), which in the Landau-Ginzburg paradigm all correspond to the same disordered phase. Then we may need to revisit the wisdom we learned from classical critical phenomena as well. Exotic QCPs beyond the classic Landau-Ginzburg-Wilson-Fisher paradigm have been extensively discussed both theoretically ${ }^{4 / 5}$ and numerically $\sqrt{6}$, including recently developed duality understanding of these QCPs $\$ 910$. Mostly recently, new possibilities of QCPs have been pointed out ${ }^{111}$ : there are generic unfine-tuned but meanwhile unnecessary (avoidable) QCPs, namely there exists a symmetry allowed route which corresponds to a strong enough deformation of the Hamiltonian in the phase diagram that smoothly connects the two stable fixed points (phases) on the two sides of the QCP, but the deformation is perturbatively irrelevant at the QCP below certain threshold. The schematic phase diagram and RG flow around the unnecessary QCP is sketched in Fig. 1.

The simplest generic unnecessary QCP examples discussed in Ref. 11 require eight massless Dirac fermions with a $\mathrm{SO}(7)$ symmetry, in both two and three spatial dimensions. This phenomenon is deeply related to the interacting topological insulators, and it was understood that interaction can reduce/collapse the classification of some of the topological insulator (TI) or topological superconductor (TSC) $)^{12} 20$. For example, the TSC ${ }^{3} \mathrm{He}-\mathrm{B}$ phase has a $\mathbb{Z}$ classification with time-reversal symmetry with $\mathcal{T}^{2}=-1$, but under time-reversal allowed interaction the classification of this TSC is reduced to $\mathbb{Z}_{16}$. This implies that for 16 copies of the TSC, the topological nontrivial and trivial phases in the noninteracting limit can be connected smoothly with strong enough local interaction. However, in the noninteracting limit, the topological-to-trivial transition of 16 copies of TSC is described by 16 massless Majorana fermions (or mathematically equivalent to 8 massless Dirac fermions), then as long as we impose an extra flavor symmetry (for example the $\mathrm{SO}(7)$ symmetry in Ref. (11) to guarantee that the 16 Majorana fermions all become massless simultaneously while the reduction of classification is still valid with the extra flavor symmetry, then this QCP in the noninteracting limit is still a generic unfine-tuned QCP, because short range interactions are irrelevant at the free 


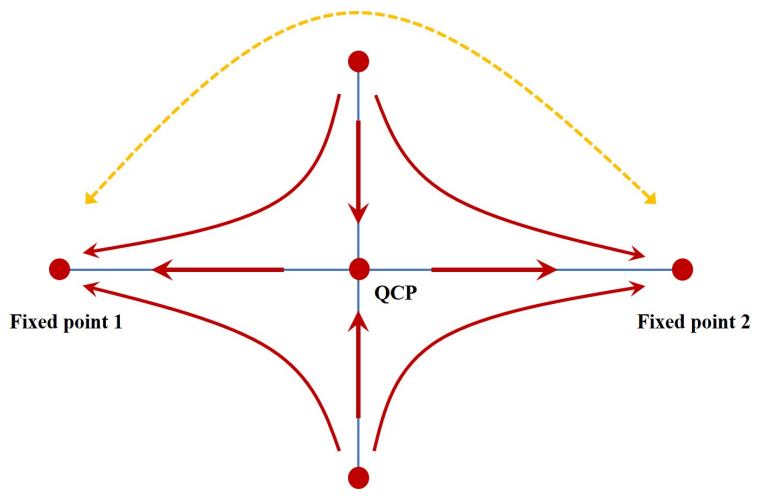

FIG. 1: The schematic phase diagram and renormalization group flow of the generic unnecessary QCP. The horizontal axis is the tuning parameter between the two phases (stable fixed points 1 and 2), in the examples discussed in this work it corresponds to the mass of either two or one $(3+1) d$ Dirac fermions; the vertical axis is the interaction, which is perturbatively irrelevant at the QCP in the noninteracting limit. There exists a adiabatic curve (dashed curve) in the entire phase diagram that connects fixed points 1 and 2 .

massless Dirac fermion fixed point for spatial dimensions higher than 1.

In this work we seek for much simpler examples of such generic unnecessary QCPs. We discuss two examples which happen in strongly interacting $3 d$ TI and $3 d$ TSC respectively. In the infrared limit, the first example is described by only two massless $(3+1) d$ Dirac fermion. In the noninteracting limit, one side of the QCP is a trivial state, while the other side is a $3 d$ topological insulator with $\mathrm{U}(1) \times Z_{n} \times \mathcal{P}$ symmetry, where $n$ is an odd integer, and $\mathcal{P}$ is a spatial-reflection. In the noninteracting limit, this TI has a $\mathbb{Z}$ classification, and the trivial-totopological transition is described by two $(3+1) d$ massless Dirac fermions. An extra time-reversal symmetry $\mathcal{T}$ with $\mathcal{T}^{2}=+1$ will guarantee that there is only one direct transition between the trivial and topological insulator phases. However, we will demonstrate that this TI can always be trivialized by local interactions, hence the QCP of the trivial-to-topological transition in the noninteracting limit can be avoided by strong enough interaction, which is perturbatively irrelevant at the QCP in the nonnteracting case.

The second example of unnecessary QCP we find is even simpler: it is formally described by only one massless Dirac fermion (two massless Majorana fermions) in the infrared limit, and one side of the QCP is a $3 d$ TSC with $\left(Z_{2 n} \rtimes Z_{4}^{T}\right) / Z_{2}$ symmetry with an odd integer $n$ (the $Z_{4}^{T}$ refers to a time-reversal symmetry with $\mathcal{T}^{2}=-1$ ), while the other side is a trivial superconductor. In the noninteracting limit, the TSC is topologically nontrivial with a classification $\mathbb{Z}_{2}$, while this TSC is again trivialized by local interactions. Hence the trivialto-topological transition is again unnecessary but generic.

\section{GENERIC UNNECESSARY QCP IN A $3 d$ TI}

\section{A. Preparation: $2 d$ TI with $\mathrm{U}(1) \times Z_{n}$ symmetry}

To construct our $3 d$ system, we need to first understand the $2 d$ TI with the $\mathrm{U}(1) \times Z_{n}$ symmetry, and we will focus on the case with odd integer $n$. This TI was discussed in Ref. $21 / 22$ in different physics contexts, and for $n=3$ this TI corresponds to the valley Chern insulator ${ }^{22}$ that is realized in Moiré systems 23 30. In this section we will review the understanding of interacting $2 d$ TI with $\mathrm{U}(1) \times Z_{n}$ symmetry. We will also impose another timereversal symmetry $\mathcal{T}$ with $\mathcal{T}^{2}=+1$, hence the entire symmetry group is $\left(\mathrm{U}(1) \rtimes Z_{2}^{T}\right) \times Z_{n}$. This time-reversal symmetry $\mathcal{T}$ is realized in the spin-polarized correlated insulator at half-filling in the miniband of the twisted double biayer graphene system $131 \underline{34}$, and $\mathcal{T}$ corresponds to an ordinary time-reversal symmetry of electrons times a spin flipping.

This TI can be naturally embedded into a nonchiral topological insulator (TI) with $\mathrm{U}(1)_{c} \times \mathrm{U}(1)_{s}$ symmetry. There are only two elementary fermions with charge $(1,1)$ and $(1,-1)$ under the $\mathrm{U}(1)_{c} \times \mathrm{U}(1)_{s}$ symmetry, and for the simplest case they form Chern insulators with Chern number \pm 1 respectively. At the free fermion level, the Hamiltonian of the $1 d$ edge state of this TI is

$$
H=\int d x \psi^{\dagger}\left(-\mathrm{i} \sigma^{3} \partial_{x}\right) \psi
$$

The symmetries act on the boundary fermions as

$$
\begin{aligned}
\mathrm{U}(1) & : \psi_{1} \rightarrow e^{\mathrm{i} \alpha} \psi_{1}, \quad \psi_{2} \rightarrow e^{\mathrm{i} \alpha} \psi_{2} \\
Z_{n} & : \psi_{1} \rightarrow e^{2 \pi \mathrm{i} / n} \psi_{1}, \quad \psi_{2} \rightarrow e^{-2 \pi \mathrm{i} / n} \psi_{2} . \\
\mathcal{T} & : \psi_{1} \rightarrow \psi_{2}, \quad \psi_{2} \rightarrow \psi_{1} .
\end{aligned}
$$

The $Z_{n}$ symmetry guarantees that no fermion bilinear mass term can be added to the boundary Hamiltonian. Also, for arbitrary copies of the TI, fermion bilinear mass operators are always forbidden. Hence the classification of this TI in the noninteracting limit is $\mathbb{Z}$.

To describe the nonchiral TI with ininteraction, we can use the $K$-matrix formalism $\frac{35}{3}$. The system can be described by the following Chern-Simons theory

$$
\mathcal{L}=\frac{\mathrm{i}}{4 \pi} \sum_{A, B=1,2} K^{A B} a^{A} \wedge d a^{B}, \quad K=\left(\begin{array}{cc}
1 & 0 \\
0 & -1
\end{array}\right),
$$

where $a^{A}$ with $A=1,2$ are two dynamical U(1) gauge fields. The edge state of this TI is described by the Luttinger liquid theory with two chiral boson fields $\phi_{1}, \phi_{2}$ and the same $K$-matrix above ${ }^{36 / 37}$ :

$$
\mathcal{L}_{\text {edge }}=\sum_{A, B=1,2} \frac{K^{A B}}{4 \pi} \partial_{x} \phi_{A} \partial_{t} \phi_{B}-\frac{V^{A B}}{4 \pi} \partial_{x} \phi_{A} \partial_{x} \phi_{B},
$$


where $V$ is a $2 \times 2$ positive-definite velocity matrix. In this theory, the boson fields satisfy the equal time commutation relation $\left[\phi_{A}(x), \partial_{y} \phi_{B}(y)\right]=2 \pi \mathrm{i}\left(K^{-1}\right)^{A B} \delta(x-y)$. Under the $\mathrm{U}(1) \times Z_{n}$ and time-reversal symmetry, the chiral boson fields $\phi_{1,2}$ transform as

$$
\begin{aligned}
\mathrm{U}(1) & : \phi_{1} \rightarrow \phi_{1}+\alpha, \quad \phi_{2} \rightarrow \phi_{2}+\alpha, \\
Z_{n}: & \phi_{1} \rightarrow \phi_{1}+\frac{2 \pi}{n}, \quad \phi_{2} \rightarrow \phi_{2}-\frac{2 \pi}{n}, \\
\mathcal{T} & : \phi_{1} \rightarrow-\phi_{2}, \quad \phi_{2} \rightarrow-\phi_{1} .
\end{aligned}
$$

Now we demonstrate that the nonchiral TI with $\mathrm{U}(1) \times$ $Z_{n}$ and the time-reversal symmetry defined above at most has a $\mathbb{Z}_{n}$ classification under local interaction, for odd integer $n$. The fact that $n$ copies of such TI together are topologically trivial can be seen from the edge theory of this system which consists of $n$ copies of Luttinger liquid theory Eq. 4. Let's denote chiral boson fields in this $n$ copy Luttinger liquid theory as $\phi_{i, A}$ where $i=1$ is the copy index and $A=1,2$ is the label for the chiral bosons within each copy. The boundary of $n$ copies of the TI can be gapped out by the following symmetric boundary interaction without ground state degeneracy:

$$
\begin{aligned}
\mathcal{L}_{\text {edge }}^{(1)} & =-\cos \left(\sum_{i=1}^{n}\left(\phi_{i, 1}-\phi_{i, 2}\right)\right) \\
& -\sum_{i=1}^{n-1} \cos \left(\phi_{i, 1}+\phi_{i, 2}-\phi_{i+1,1}-\phi_{i+1,2}\right) .
\end{aligned}
$$

These are local interacting terms between the electrons, and they preserve all the symmetries.

This edge theory $\mathcal{L}_{\text {edge }}^{(1)}$ can be analyzed systematically as following: There are in total $n$ different terms in $\mathcal{L}_{\text {edge }}^{(1)}$, and we can represent each term in $\mathcal{L}_{\text {edge }}^{(1)}$ as $\cos \left(\boldsymbol{\Lambda}_{I} \cdot \boldsymbol{\Phi}\right) . \boldsymbol{\Lambda}_{I}$ are $2 n$ component vectors $(I=1, \cdots n)$, and $\boldsymbol{\Phi}=\left(\phi_{1,1}, \phi_{1,2}, \phi_{2,1} \cdots\right) . \boldsymbol{\Lambda}_{I}$ are a set of minimal linearly independent integer vectors, and they satisfy the condition $\underline{38139}$

$$
\boldsymbol{\Lambda}_{I}^{t} \mathbf{K}^{-1} \boldsymbol{\Lambda}_{J}=0
$$

for any $I, J=1, \cdots n$. Here $\mathbf{K}$ is the $2 n \times 2 n$ blockdiagonal $K$-matrix for $n$ copies of the nonchiral TI with $\mathrm{U}(1) \times Z_{n}$ symmetry. Eq. 7 implies that the arguments in all the cosine terms in $\mathcal{L}_{\text {edge }}^{(1)}$ commute with each other, and hence all terms in $\mathcal{L}_{\text {edge }}^{(1)}$ can be minimized simultaneously, which leads to a fully gapped edge state.

The $2 n$-component integer vector $\boldsymbol{\Lambda}_{I}$ is a vector in a $2 n$ dimensional cubic lattice with lattice constant 1 . Linear combinations of $\boldsymbol{\Lambda}_{I}$ span a $n$-dimensional hyperplane of this $2 n$ dimensional cubic lattice. To be rigorous we also need to show that $\boldsymbol{\Lambda}_{I}$ are the irreducible basis vectors of the lattice sites residing on this $n$-dimensional hyperplane, hence the minimum of $\mathcal{L}_{\text {edge }}^{(1)}$ has no degeneracy. This can also be verified for odd integer $n$.
Having shown that $n$ copies of the nonchiral TI with $\left(\mathrm{U}(1) \rtimes Z_{2}^{T}\right) \times Z_{n}$ symmetry together is topologically trivial, we now argue that the classification of such nonchiral TI has to be $\mathbb{Z}_{n}$, with odd integer $n$. For $k$ copies of such nonchiral TI, we can consider, in the non-interacting limit, $k$ copies of the edge theory Eq. 1 residing on a circle. Every time a $2 \pi$ flux associate to the U(1) symmetry is threaded through the circle, the total $Z_{n}$ charge of this $k$-copy edge theory is shifted by $2 k$. Given that $Z_{n}$ charges are defined modulo odd integer $n$, if the number of copies $k$ is not a multiple of $n$, the shift of $Z_{n}$ charge on the edge is non-trivial indicating that the edge theory is in fact anomalous and further suggesting that its associated bulk is topologically non-trivial. Therefore, $n$ is the "minimal number" of copies needed for the edge to be non-anomalous. Combined with the previous argument, the classification of the nonchiral TI with $\left(\mathrm{U}(1) \rtimes Z_{2}^{T}\right) \times Z_{n}$ symmetry has to be $\mathbb{Z}_{n}$.

For odd integer $n$, a general connection between the nonchiral TI with $\left(\mathrm{U}(1) \rtimes Z_{2}^{T}\right) \times Z_{n}$ symmetry and bosonic symmetry protected topological (bSPT) state with the same symmetry ${ }^{\sqrt{213}}$ can be made. Since the interacting TI has a $\mathbb{Z}_{n}$ classification, one copy of the elementary TI is topologically equivalent to $n+1$ (an even integer) copies of the TI; while according to Ref. 40 43, even number of such nonchiral TIs can be "glued" into a bSPT state with the same symmetry under interaction, where all the local fermion excitations at the boundary are gapped out by interaction, leaving only symmetry protected gapless local bosonic excitations.

A variety of bSPT states and their edge states can be described by the Chern-Simons theory with the following $K$-matrix ${ }^{44}$, whose boundary state is described by two chiral bosons $\varphi$ and $\theta$ with $K$-matrix:

$$
K_{\mathrm{bSPT}}=\left(\begin{array}{ll}
0 & 1 \\
1 & 0
\end{array}\right) .
$$

The chiral bosons transform under the symmetries as

$$
\begin{aligned}
\mathrm{U}(1) & : \varphi \rightarrow \varphi+2 \alpha, \quad \theta \rightarrow \theta \\
Z_{n} & : \varphi \rightarrow \varphi, \quad \theta \rightarrow \theta-2 \pi / n, \\
\mathcal{T} & : \varphi \rightarrow-\varphi, \quad \theta \rightarrow \theta .
\end{aligned}
$$

Now, we consider a $(1+1) d$ interface between the bSPT and the nonchiral TI discussed previously which can be described by the four boson fields $\phi_{1,2}, \varphi$ and $\theta$. The symmetry allowed interaction that can gap out this interface without degeneracy is

$$
\mathcal{L}_{\text {edge }}^{(2)} \sim-\cos \left(\phi_{1}+\phi_{2}-\varphi\right)-\cos \left(\phi_{1}-\phi_{2}+2 \theta\right) .
$$

Again the arguments in the cosine terms commute with each other, hence all terms in $\mathcal{L}_{\text {edge }}^{(2)}$ can be minimized simultaneously, and the interface is gapped out without degeneracy through the same reasoning as before. The existence of such a gapped interface between the bSPT 
and the fermionic TI guarantees the topological equivalence between the two sides of this interface.

The physical interpretation of the bosonoic fields $\varphi$ and $\theta$ can be understood in terms of their quantum numbers. The local boson field $e^{\mathrm{i} \varphi}$ can be identified with the bound state $\psi_{1} \psi_{2}$; the quantum number of the single boson operator $e^{\mathrm{i} \theta}$ is equivalent to $(n+1) / 2$ copies of the particlehole pair $\psi_{1}^{\dagger} \psi_{2}\left(Z_{n}\right.$ charge is defined $\left.\bmod n\right)$. We can see that when and only when $n$ is an odd integer, $e^{\mathrm{i} \theta}$ can be viewed as a local boson field. Hence for odd integer $n$, a nonchiral TI with $\mathrm{U}(1) \times Z_{n}$ symmetry and time-reversal $\mathcal{T}$ with $\mathcal{T}^{2}=+1$ is equivalent to a bSPT constructed with local bosons.

This result implies that, under interaction the $2 d$ nonchiral TI with $\left(\mathrm{U}(1) \rtimes Z_{2}^{T}\right) \times Z_{n}$ symmetry can have fully gapped single electron excitation, but meanwhile symmetry protected gapless local boson excitations at its boundary. This was thought to be only possible for even copies of nonchiral TI such as the quantum spin Hall insulator with spin $S^{z}$ conservation $\underline{40}$.

\section{B. Generic unnecessary QCP in two dimensions}

The result in the previous section is sufficient to predict a generic unnecessary QCP in $2 d$. In the noninteracting limit, the topological transition between the trivial state and the nonchiral TI with $\left(\mathrm{U}(1) \rtimes Z_{2}^{T}\right) \times Z_{n}$ symmetry is described by two massless $(2+1) d$ two-component Dirac fermions. The time-reversal symmetry $\mathcal{T}$ guarantees that there is a single transition between the trivial and topological state. Now if we consider $n$ copies of the TIs, with extra assumptions of a discrete cyclic symmetry between the $n$ copies of TIs, and also a fermion parity of each copy of the TI, a single generic direct trivialto-topological transition of $n$ copies of the TIs is still guaranteed, which is described by $2 n$ massless $2 d$ Dirac fermion.

One can check that the interaction Lagrangian Eq. 6 also preserves the extra cyclic symmetry and fermion parity of each copy, then $n$ copies of the TI is still trivialized by interaction. Though short range interaction is irrelevant at the $(2+1) d$ Dirac fermion, the generic direct trivial-to-topological transition of $n$-copies of the TI described above becomes unnecessary under strong enough symmetry-allowed local interaction. The minimum situation we find in $2 d$ would be $n=3$, which may have been realized as the spin polarized correlated insulator in the twisted double bilayer graphene 31 .

In this section we demonstrate that the generic unnecessary QCP can be even simpler in $3 d$. In the noninteracting limit, the topological transition between the trivial state and the nontrivial $3 d$ TI with $\mathrm{U}(1) \times Z_{n} \times \mathcal{P}$ symmetry is described by two massless $(3+1) d$ four-component Dirac fermions. But we will demonstrate that this transition is unnecessary under interaction.

\section{Noninteracting $3 d$ TI with $\mathrm{U}(1) \times Z_{n} \times \mathcal{P}$ symmetry}

Now we switch gear to the $3 d$ TI with $\mathrm{U}(1) \times Z_{n} \times \mathcal{P}$ symmetry. The $2 d$ boundary state of this TI is described by the Hamiltonian

$$
H_{\text {edge }}=\int d^{2} x \psi^{\dagger}\left(\mathrm{i} \sigma^{10} \partial_{x}+\mathrm{i} \sigma^{33} \partial_{y}\right) \psi
$$

$\sigma^{a b}=\sigma^{a} \otimes \sigma^{b}$. The symmetries act on the $2 d$ boundary fermions as

$$
\begin{aligned}
\mathrm{U}(1) & : \psi \rightarrow e^{i \alpha} \psi, \\
\mathcal{P} & : x \rightarrow-x, \quad \psi \rightarrow \sigma^{30} \psi, \\
Z_{n} & : \psi \rightarrow \exp \left(\mathrm{i} \frac{2 \pi}{n} \sigma^{03}\right) \psi .
\end{aligned}
$$

One can check that all the mass terms at this $2 d$ boundary, such as

$$
\psi^{\dagger} \sigma^{20} \psi, \quad \psi^{\dagger} \sigma^{23} \psi, \quad \psi^{\dagger} \sigma^{31} \psi, \quad \psi^{\dagger} \sigma^{32} \psi
$$

are forbidden by either the reflection, or the $Z_{n}$ symmetry. We can also add another time-reversal symmetry $\mathcal{T}$ with $\mathcal{T}^{2}=+1$ :

$$
\mathcal{T}: \psi \rightarrow \sigma^{31} \psi
$$

Also, one can check that for arbitrary copies of the system, all the fermion bilinear mass terms are still forbidden by either the $\mathcal{P}$ or $Z_{n}$ symmetry, hence the classification of this $3 d$ TI in the free fermion limit is $\mathbb{Z}$.

At the free fermion level, the bulk trivial-to-topological phase transition of a single copy of this $3 d \mathrm{TI}$ is described by the following Hamiltonian:

$$
\begin{aligned}
H_{\mathrm{bulk}} & =\int d^{3} x \psi^{\dagger}\left(\mathrm{i} \sigma^{103} \partial_{x}+\mathrm{i} \sigma^{333} \partial_{y}+\mathrm{i} \sigma^{002} \partial_{z}\right) \psi \\
& +m \psi^{\dagger} \sigma^{001} \psi
\end{aligned}
$$

Now $\psi$ becomes an eight component fermion. All the symmetries act on the bulk fermions as

$$
\begin{gathered}
\mathcal{P}: \psi \rightarrow \sigma^{300} \psi, \quad \mathcal{T}: \psi \rightarrow \sigma^{310} \psi \\
\mathrm{U}(1): \psi \rightarrow e^{i \alpha} \psi, \quad Z_{n}: \psi \rightarrow \exp \left(\mathrm{i} \frac{2 \pi}{n} \sigma^{030}\right) \psi
\end{gathered}
$$

One can check that, at the $x y 2 d$ boundary of the system, the bulk Hamiltonian Eq. 14 reduces to the $2 d$ boundary Hamiltonian Eq. 10, which corresponds to a domain wall of $m$ long the $\hat{z}$ axis in Eq. 14 , and all the bulk symmetry actions reduce exactly to the symmetry actions on the boundary defined above.

There are other mass matrices in the $3 d$ bulk:

$$
\sigma^{203}, \quad \sigma^{233}, \quad \sigma^{313}, \quad \sigma^{323}, \quad \sigma^{121}, \quad \sigma^{111}, \quad \sigma^{031} .
$$




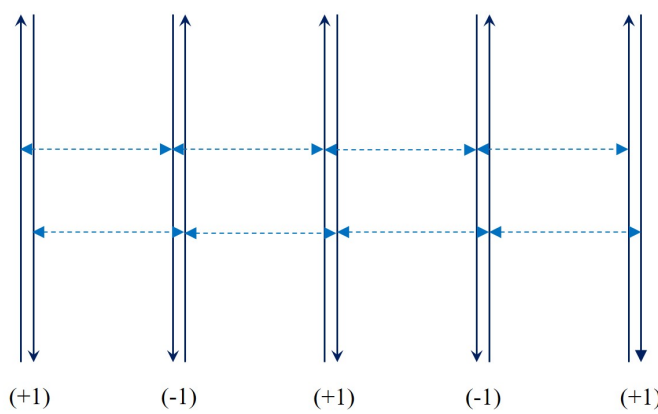

FIG. 2: The coupled wire construction of the $2 d$ edge state Hamiltonian Eq. 10 of the noninteracting $3 d$ TI with $\mathrm{U}(1) \times$ $Z_{n} \times \mathcal{P}$ symmetry. Each wire represents the $1 d$ edge state of a $2 d$ TI with $\mathrm{U}(1) \times Z_{n}$ symmetry, which is described by Eq.1 Each wire is composed of a pair of counter-propagating $1 d$ fermion modes that carry opposite $Z_{n}$ charges. Symmetry allowed tunnellings between the wires will drive the system into the $2 d$ edge state Hamiltonian Eq. 10.

All these extra mass terms are forbidden, one way or another. The most interesting, and probably important "extra" mass term is the last one: $\psi^{\dagger} \sigma^{031} \psi$. This mass term, if exists, does not gap out the transition, but split the transition into two. But this extra mass term is forbidden by $\mathcal{T}$. So $\mathcal{T}$ is the symmetry that guarantees a generic direct single trivial-to-topological transition at the free fermion level. But later we will show that this transition will be avoided under interaction.

\section{Interacting TI and Unnecessary QCP in three dimensions}

In Ref. 45, a general approach of understanding and constructing $3 d$ symmetry protected topological (SPT) state (generalization of topological insulator) with a reflection symmetry was proposed. To construct a $3 d$ SPT state with reflection $\mathcal{P}$, one can start with a $2 d$ system on the reflection-invariant plane. The $3 d$ SPT state can always be constructed by stacking layers of $2 d$ SPT states on the reflection plane. But even when the $2 d$ SPT state is a nontrivial SPT state, it does not guarantee that the $3 d$ SPT state is nontrivial, more detailed analysis of the procedure of stacking is demanded.

The $2 d$ boundary state of the $3 d$ system can then be constructed by stacking the $1 d$ (wire) boundary state of the $2 d$ layer SPT states. This construction is often referred to as the coupled wire construction. Two sides of the $1 d$ wire are connected by reflection $x \rightarrow-x$, hence in the noninteracting limit this wire can be viewed as the domain wall of the mass term $m(x) \psi^{\dagger} \sigma^{20} \psi$ at the $2 d$ boundary. The coupled wire construction of the boundary states can be viewed as coupling the domain wall states with oscillating sign of $m$ along the $\hat{x}$ direction, and the domain walls are along the $\hat{y}$ direction. Let us assume that each domain has width 1 . At the domain

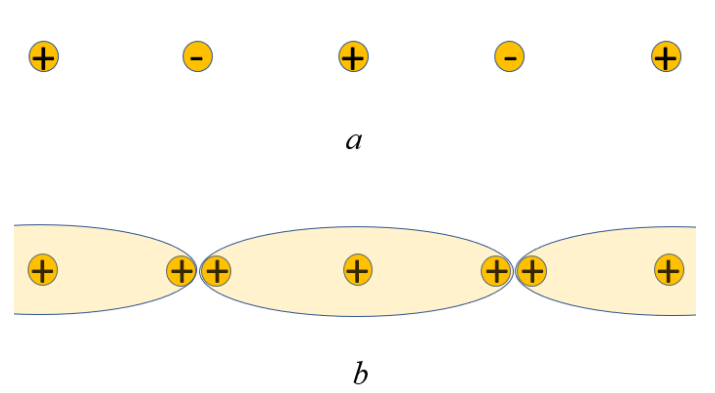

FIG. 3: The coupled wire construction for the symmetric gapped edge state with $n=3$. Each wire is the boundary state of a $2 d$ layer, and the wire with index $(-1)$ can be adiabatically deformed into a wire with index $(+2)$ under interaction without any transition in the $2 d$ layer, due to the $\mathbb{Z}_{3}$ classification of the layer. Hence the wires can be regrouped and gapped out by interactions that preserves all the symmetries.

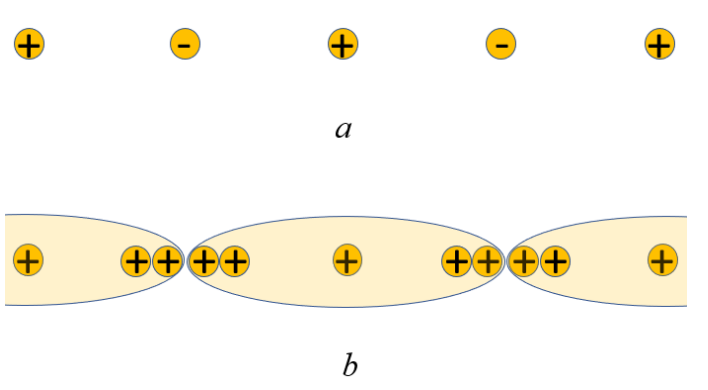

FIG. 4: Illustration of the symmetric gapped edge state with $n=5$. Each wire with index $(-1)$ can be deformed into a wire with index $(+4)$ under interaction, and again the wires can be regrouped and gapped out by interactions that preserve all the symmetries.

wall of $m(x)$, the domain wall wave functions are eigenstates of $\sigma^{30}=(-1)^{x}$ for every other wire.

Since the wire states have eigenvalues $\sigma^{30}= \pm 1$, the Hamiltonian and the symmetries project on the wire states as the following:

$$
\begin{aligned}
H(x)_{\text {wire }} & =\int d y(-1)^{x} \psi^{\dagger} \mathrm{i} \sigma^{3} \partial_{y} \psi, \\
\mathcal{P} & : \psi(x) \rightarrow(-1)^{x} \psi(-x), \quad \mathcal{T}: \psi \rightarrow(-1)^{x} \sigma^{1} \psi \\
Z_{n} & : \psi \rightarrow \exp \left(\mathrm{i} \frac{2 \pi}{n} \sigma^{3}\right) \psi .
\end{aligned}
$$

By turning on tunnellings between the wires, one can exactly reproduce the free fermion Hamiltonian of the $2 d$ boundary state Eq. 10 .

$$
\begin{aligned}
H_{\text {edge }} & =\sum_{x} \int d y(-1)^{x} \psi^{\dagger} \mathrm{i} \sigma^{3} \partial_{y} \psi+\mathrm{i} t \psi_{x}^{\dagger} \sigma^{0} \psi_{x+1}+\text { H.c. } \\
& \sim \int d^{2} x \psi^{\dagger}\left(\mathrm{i} t \sigma^{10} \partial_{x}+\mathrm{i} \sigma^{33} \partial_{y}\right) \psi .
\end{aligned}
$$

An extra Pauli space emerges in the low energy theory because there are two wires per unit cell in this wire 
construction. Here we turned on a uniform tunnelling between neighboring wires. If instead a staggered tunnelling $\sum_{x}(-1)^{x} \mathrm{i} t^{\prime} \psi_{x}^{\dagger} \sigma^{0} \psi_{x+1}+H . c$. is turned on, the reflection symmetry will be broken and a mass term $\psi^{\dagger} \sigma^{20} \psi$ will be generated at low energy.

However, by turning on interaction, one can show that the entire system is trivialized, even for a single copy of the $3 d$ system. In the following we will demonstrate this with $n=3$, but this argument can be generalized to any odd integer $n$. Let us give index $(+1)$ to a $1 d$ wire described by one pair of counter-propagating $1 d$ fermion modes, with fermion carrying charge \pm 1 under symmetry $Z_{3}$ moving along the $\pm \hat{y}$ direction along the wire. First of all, quoting the results from the previous section, each layer of the $2 d$ SPT state has a $\mathbb{Z}_{3}$ classification, hence each wire with index $( \pm 1)$ can be deformed continuously through interaction into two pairs of counterpropagating $1 d$ fermion modes, and the fermions carrying $\pm 1 Z_{3}$ charges move along the $\mp \hat{y}$ direction. Or in other words a wire with index $( \pm 1)$ can be continuously deformed in to a wire carrying index $(\mp 2)$ through interaction.

Now again, using the fact that the $2 d$ interacting TI with $\left(\mathrm{U}(1) \rtimes Z_{2}^{T}\right) \times Z_{3}$ has a $\mathbb{Z}_{3}$ classification, we can group the wires as Fig. $3 b$, and turn on inter-wire interaction to gap out states along the wires, and we can see that this arrangement preserves all the symmetries including $\mathcal{P}$ and $\mathcal{T}$, and even translation symmetry. This means that under interaction which preserves all the symmetries, the boundary state of the $3 d$ TI can be gapped out by interaction, hence even a single copy of the $3 d$ TI is trivialized by interaction. The same construction can be generalized to other odd integer $n$, for example the case with $n=5$ is illustrated in Fig. $4 p$.

Now because the TI is trivialized by interaction, the bulk direct trivial-to-topological transition in the noninteracting limit, which is described by two massless $(3+1) d$ Dirac fermions, becomes "unnecessary" under strong enough interaction.

\section{E. Interacting TI: mapping to bosonic system}

Using the chiral boson language, we can still show that each wire is equivalent to the edge state of a $2 d$ bosonic symmetry protected topological (bSPT) state, which can be shown by coupling each wire to the boundary of a $2 d$ bSPT state. We can still describe this entire coupled $1 d$ system using chiral bosons, and this $1 d$ system can be symmetrically gapped by the same Lagrangian as $\mathcal{L}_{\text {edge }}^{(2)}$ Eq. 9. The $\mathcal{P}, \mathcal{T}$ symmetries act on all the chiral boson fields as

$$
\begin{gathered}
\mathcal{P}: \phi_{1,2} \rightarrow \phi_{1,2}+\frac{1+(-1)^{x}}{2} \pi, \\
\varphi \rightarrow \varphi, \quad \theta \rightarrow \theta ; \\
\mathcal{T}: \phi_{1,2} \rightarrow-\phi_{2,1}+\frac{1+(-1)^{x}}{2} \pi,
\end{gathered}
$$

$$
\varphi \rightarrow-\varphi, \quad \theta \rightarrow \theta
$$

$\exp (\mathrm{i} \varphi)$ and $\exp (\mathrm{i} \theta)$ are still local bosons for odd integer $n$, i.e. they are bound state of local fermions.

There are standard formalisms of describing and constructing the potentially nontrivial $3 d$ bSPT state, such as the group cohomology $\sqrt{213}$, and the effective nonlinear sigma model with a topological $\Theta-$ term 46 48. The bulk bosonic state can be described by the action

$$
\begin{aligned}
\mathcal{S}_{\text {bulk }} & =\int d^{3} x d \tau \frac{1}{g}\left(\partial_{\mu} \boldsymbol{n}\right)^{2} \\
& +\frac{\Theta \mathrm{i}}{\Omega_{4}} \epsilon_{a b c d e} n^{a} \partial_{x} n^{b} \partial_{y} n^{c} \partial_{z} n^{d} \partial_{\tau} n^{e},
\end{aligned}
$$

where $\Theta=2 \pi, \Omega_{4}$ is the volume of a four-dimensional sphere with unit radius. The unit-length five-component vector field $\boldsymbol{n}(\boldsymbol{r}, \tau)$ can be parameterized as

$$
\begin{aligned}
\left(n_{1}, n_{2}\right) & =\cos (\beta) \cos (\gamma)(\cos (\varphi), \sin (\varphi)), \\
\left(n_{3}, n_{4}\right) & =\cos (\beta) \sin (\gamma)(\cos (\theta), \sin (\theta)), \\
n_{5} & =\sin (\beta) ;
\end{aligned}
$$

Under symmetries, the vector $\boldsymbol{n}(\boldsymbol{r}, \tau)$ transform as

$$
\begin{aligned}
\mathrm{U}(1) & : \varphi \rightarrow \varphi+2 \alpha, \quad \theta \rightarrow \theta, \quad \beta \rightarrow \beta \\
Z_{n} & : \varphi \rightarrow \varphi, \quad \theta \rightarrow \theta-2 \pi / n, \quad \beta \rightarrow \beta \\
\mathcal{T} & : \varphi \rightarrow-\varphi, \quad \theta \rightarrow \theta, \quad \beta \rightarrow \beta \\
\mathcal{P} & : \varphi \rightarrow \varphi, \quad \theta \rightarrow \theta, \quad \beta \rightarrow-\beta .
\end{aligned}
$$

Hence $n_{5}$ is invariant under $\left(\mathrm{U}(1) \rtimes Z_{2}^{T}\right) \times Z_{n}$ symmetry, but odd under reflection $\mathcal{P}$. The action Eq. 20 is invariant under all the symmetries.

Based on the CPT theorem, we can replace a field theory with $\mathcal{P}$ and $\mathcal{T}$ symmetry, by the $\mathcal{C}$ and $\mathcal{T}$ symmetry, and $\mathcal{C}$ acts on the fields as

$$
\mathcal{C}: \varphi \rightarrow-\varphi, \quad n_{5} \rightarrow-n_{5} .
$$

Thus we can view $b \sim \cos (\varphi)+\mathrm{i} \sin (\varphi)$ as a bosonic rotor field, and $n_{5}$ is the density of the boson. And $\mathcal{C}$ is the particle-hole transformation of the bosonic rotor field. Now all the symmetries are internal symmetries of the field theory Eq. 20.

Now as a consistency check we need to show that the bSPT is actually trivial. This can be shown using the same method as Ref. 49. We can first embed the $Z_{n}$ symmetry into another $\mathrm{U}(1)_{s}$ symmetry, and $\cos (\theta)+$ $\mathrm{i} \sin (\theta)$ becomes a rotor under the $\mathrm{U}(1)_{s}$ symmetry. Then the $3 d$ bSPT can be understood using the "decorated vortex" picture. If we consider a vortex of line of $\theta$ (the vortex configuration preserves the $\mathrm{U}(1), Z_{2}^{T}$, and $\mathcal{C}$ ), the action Eq. 20 is reduced to the following $(1+1) d$ NLSM defined with a three component unit vector $\tilde{\boldsymbol{n}}(x, \tau)$ :

$$
\mathcal{S}_{1 \mathrm{~d}}=\int d x d \tau \frac{1}{g}\left(\partial_{\mu} \tilde{\boldsymbol{n}}\right)^{2}+\frac{\Theta \mathrm{i}}{4 \pi} \epsilon_{a b c} \tilde{n}^{a} \partial_{x} \tilde{n}^{b} \partial_{\tau} \tilde{n}^{c},
$$


again $\Theta=2 \pi$. The three component vector $\tilde{\boldsymbol{n}}$ is

$$
\tilde{\boldsymbol{n}}=(\cos (\beta) \cos (\varphi), \cos (\beta) \sin (\varphi), \sin (\beta)) .
$$

This implies that the vortex of $\mathrm{U}(1)_{s}$ symmetry is decorated with a $1 d$ Haldane phase of $\tilde{\boldsymbol{n}}^{50}$, which is known to have a $\mathbb{Z}_{2}$ classification. Thus the $3 d$ bSPT state can be constructed by first spontaneously breaking the $\mathrm{U}(1)_{s}$ symmetry in the $3 d$ bulk (developing a superfluid phase), then decorate the vortex loop of this superfluid phase with the Haldane phase described above, and then proliferate/condense the decorated vortex loop.

Once we break $\mathrm{U}(1)_{s}$ down to $Z_{n}$ with odd integer $n$, this decorated vortex picture will yield a trivial $3 d$ bulk state. This can be perceived by the fact that the $Z_{n}$ vortex loop has a " $\mathbb{Z}_{n}$ classification", i.e. the $n$-copies of $Z_{n}$ vortex loop is a trivial configuration in the spacetime. The $\mathbb{Z}_{n}$ classification with odd $n$ is incompatible with the $\mathbb{Z}_{2}$ classification of the Haldane phase decorated on the vortex loop.

More explicitly, one can demonstrate that the $2 d$ boundary of Eq. 20 can be symmetrically gapped out without any degeneracy with odd integer $n$. Again, let us start with the $\mathrm{U}(1)_{s}$ symmetry, a single vortex of $\mathrm{U}(1)_{s}$ on the $2 d$ boundary is the termination of the vortex line in the bulk, which carries the $0 d$ boundary state of the Haldane phase discussed above, and due to the $\mathbb{Z}_{2}$ classification of the Haldane phase, a double vortex of $\mathrm{U}(1)_{s}$ will carry trivial quantum number, and hence can condense without breaking any symmetry.

After condensing the double vortex, the $2 d$ boundary becomes a $Z_{2}$ topological order, whose bosonic $e$ and $m$ anyon excitations carry fractional quantum numbers. The $e$ excitation is the remnant of the single vortex of $\mathrm{U}(1)_{s}$ symmetry after condensing the double vortices, which carries a projective representation of $\mathrm{U}(1) \rtimes Z_{2}^{T}$ and $\mathrm{U}(1) \rtimes \mathcal{C}$. Here we pay particular attention to the $m$ excitation, which should carry half-charge of $\mathrm{U}(1)_{s}$, or half-charge of $Z_{n}$ if we break $\mathrm{U}(1)_{s}$ down to its subgroup $Z_{n}$. Hence under the $Z_{n}$ transformation, the $m$ excitation acquires a phase factor

$$
Z_{n}: \Psi_{m} \rightarrow \exp \left(\mathrm{i} \frac{2 \pi}{2 n}\right) \Psi_{m}
$$

Now consider a $n$-body bound state of $\Psi_{m}$, let us denote it as $B \sim\left(\Psi_{m}\right)^{n}$. Under the $Z_{n}$ symmetry, $B$ transforms as $B \rightarrow-B$. And because $n$ is an odd integer, $B \sim\left(\Psi_{m}\right)^{n}$ still carries $Z_{2}$ gauge charge-1, and the $Z_{n}$ transformation can be cancelled by a $Z_{2}$ gauge transformation. $B$ can also be viewed as the bound state between a single $\Psi_{m}$ and $(n-1) / 2$ copies of the local boson $e^{\mathrm{i} \theta}$. Condensing $B$ at the $2 d$ boundary does not break any symmetry, and it confines the nontrivial anyons, hence the boundary is driven into a fully gapped symmetric state without degeneracy. This completes the argument that the bosonic SPT state is actually trivial, which is consistent with our conclusions in the previous subsections.

\section{GENERIC UNNECESSARY QCP IN A $3 d$ TSC}

An insulator has electron number conservation, hence it must have a $\mathrm{U}(1)$ symmetry; while a superconductor breaks the particle number conservation. In this section we discuss a superconductor with $\left(Z_{2 n} \rtimes Z_{4}^{T}\right) / Z_{2}$ symmetry, with an odd integer $n>1$. First of all, let us clarify the notation. The $Z_{4}^{T}$ stands for a time-reversal symmetry with $\mathcal{T}^{2}=-1, \mathcal{T}^{4}=+1$. But we need to mod out the common $Z_{2}$ subgroup of both $Z_{2 n}$ and $Z_{4}^{T}$. At the free fermion level, there is a nontrivial TSC with such symmetry, whose $2 d$ edge state Hamiltonian and the symmetry transformation is

$$
\begin{aligned}
H_{\text {edge }} & =\int d^{2} x \psi^{\dagger}\left(\mathrm{i} \sigma^{1} \partial_{x}+\mathrm{i} \sigma^{3} \partial_{y}\right) \psi, \\
Z_{2 n} & : \psi \rightarrow \exp \left(\mathrm{i} \frac{2 \pi}{2 n}\right) \psi, \\
\mathcal{T} & : \psi \rightarrow \mathrm{i} \sigma^{2} \psi
\end{aligned}
$$

Apparently with these symmetries no fermion bilinear term can be turned on at the boundary Hamiltonian which gaps out the boundary spectrum for any integer $n>1$. Hence at the free fermion level, Eq. 27 describes the boundary state of a nontrivial TSC.

Our goal is to study the fate of this TSC under interaction. The techniques we used in the previous section, i.e. the coupled wire construction, is no longer obviously applicable to this case. But apparently this TSC can be embedded into the TI in the AII class with $\left(\mathrm{U}(1) \rtimes Z_{4}^{T}\right) / Z_{2}$ symmetry, we can study the interaction effect by starting with the $2 d$ boundary topological order of the AII TI constructed in Ref. 53 56. This boundary topological order is anomalous with the U(1) symmetry of the AII class of TI, but we will show that this topological order becomes nonanomalous and hence can be driven into a fully symmetric gapped nondegenerate state, once $\mathrm{U}(1)$ is broken down to $Z_{2 n}$.

According to (for example) Ref. 56, the boundary of the AII TI can be driven into a topological order with in total 48 anyons (not including the electron itself). This topological order can be constructed by first driving the boundary into a superconductor by condensing Cooper pair $\psi^{t} \sigma^{2} \psi$, which spontaneously breaks the U(1) symmetry down to its $Z_{2}$ subgroup (the fermion parity of the electrons). Then the symmetries an be restored by condensing the $8 \pi$ vortex of the superconductor, i.e. eight fold bound state of the elementary vortex of the superconductor, and the $8 \pi$ vortex is a boson. Within these anyons there is a charge $1 / 4$ boson $b$, which can be viewed as a $1 / 8$ "parton" of the Cooper pair. Now if we break the $\mathrm{U}(1)$ down to $Z_{2 n}$ symmetry with odd integer $n$, under the $Z_{2 n}$ transformation, this boson transforms as

$$
Z_{2 n}: b \rightarrow \exp \left(\mathrm{i} \frac{2 \pi}{8 n}\right) b .
$$

$b$ is also coupled to a gauge field, and under the gauge 
transformation,

$$
\text { Gauge }: b \rightarrow \exp \left(\mathrm{i} \frac{k \pi}{4}\right) b
$$

with any integer $k$.

Now let us form a bound state of $b$ and $\left(n^{2}-1\right) / 8$ copies of Cooper pair $\psi^{t} \mathrm{i} \sigma^{2} \psi$ :

$$
B \sim b \times\left(\psi^{t} \mathrm{i} \sigma^{2} \psi\right)^{\left(n^{2}-1\right) / 8},
$$

notice that for odd integer $n>1, n^{2}-1$ is always an integer multiple of 8 . Then under the $Z_{2 n}$ symmetry, $B$ transforms as

$$
\begin{aligned}
Z_{2 n}: B & \rightarrow \exp \left(\mathrm{i} \frac{2 \pi}{8 n}+\mathrm{i} 2 \times \frac{2 \pi}{2 n} \times \frac{n^{2}-1}{8}\right) B \\
& =\left(\mathrm{i} \frac{n \pi}{4}\right) B .
\end{aligned}
$$

This implies that the symmetry transformation on $B$ can be cancelled by a gauge transformation. $b$ and hence $B$ are both invariant under time-reversal.

Also, Ref. 56 demonstrated that $B$ has nontrivial statistics with many of the anyons including the nonabelian Ising anyon ( $B$ does not carry any gauge independent global quantum numbers, and it carries the same gauge charge as $b$, because Cooper pairs are gauge neutral). This implies that condensing $B$ would preserve all the symmetries, and confine all the nontrivial anyons, i.e. the condensate of $B$ is a fully gapped symmetric $2 d$ boundary state without ground state degeneracy. In the condensate of $B, b$ can be identified as multiples of Cooper pair $\left(\psi^{t} \mathbf{i} \sigma^{2} \psi\right)^{\left(n^{2}-1\right) / 8}$.

There is also one deconfined neutral fermion $f$ that braids trivially with boson $B$ and $b$, but this fermion $f$ is not a fractionalized anyon in the condensate of $B$. $f$ can be identified as the bound state of the original local fermion $\psi$ and multiple of $t^{56}$. Then when we break $\mathrm{U}(1)$ down to $Z_{2 n}$ with odd integer $n$, in the condensate of $B, f$ can be viewed as the bound state of $\psi$ and multiple of Cooper pairs, which is also a Kramers doublet local fermion. This implies that, once we break U(1) to $Z_{2 n}$ with odd integer $n$, interaction trivializes the TSC. Then the bulk trivial-to-topological transition, which at the free fermion level is formally described by a single massless Dirac fermion, becomes a generic unnecessary QCP.

\section{DISCUSSION}

In this work we propose two simple examples of generic unnecessary QCPs, which are respectively described by two and one massless $(3+1) d$ Dirac fermions, while the previously known examples involve at least eight Dirac fermions. This result is based on our analysis of classification of interacting $3 d \mathrm{TI}$ and TSC. In both examples we demonstrated that the systems are topological nontrivial without interaction, but are both totally trivialized by local interactions. Local interaction is perturbatively irrelevant at the noninteracting $(3+1) d$ Dirac fermion fixed point, but a continuous route exists in the phase diagram with strong enough interaction that connects the trivial and topological phase of the TI and TSC in the noninteracting limit (Fig. 11).

The $2 d$ boundary Hamiltonian Eq. 10 of the $3 d$ TI in our first example, as well as the transformation of the fermions under symmetries are identical to the low energy theory of spinless fermion at half-filling on the honeycomb lattice with dominant nearest neighbor hopping. The fermion modes with eigenvalue $\sigma^{03}= \pm 1$ correspond to the Dirac fermion cones expanded at the two valleys of the Brillouin zone of the honeycomb lattice. The $Z_{3}$ symmetry can be viewed as the translation of the honeycomb lattice, and the reflection $\mathcal{P}$ exchanges the $\mathrm{A}$ and $\mathrm{B}$ sublattices of the honeycomb lattice. Our result supports that there exists a fully gapped and symmetric state for interacting spinless fermions on the honeycomb lattice, i.e. there is no Lieb-Shultz-Matthis $57 / 591$ like theorem for spinless fermions on the honeycomb lattice at half-filling with translation and reflection symmetry, while this is only possible under strong enough interaction.

There is another potentially interesting extension of our first example. The trivial-to-topological transition of many bSPT systems in $3 d$, can be described by a $(3+1) d \mathrm{QCP}$ with a dynamical $\mathrm{SU}(2)$ gauge field coupled with two flavors of Dirac fermions 9 . This theory has a maximal $\mathrm{SO}(5)$ global symmetry. Breaking the $\mathrm{SO}(5)$ down to our symmetries would permit more local quartic fermion terms in the Lagrangian. The original trivialto-topological transition of the bSPT system is definitely "unnecessary" because we know that this bSPT is trivial once we break the $\mathrm{SO}(5)$ down to the symmetries considered here. There is a possibility that this transition is also a generic QCP which corresponds to a strongly interacting conformal field theory. If this is the case, then the phase diagram Fig. 1 is even richer: there are two generic unnecessary QCPs in the same phase diagram, but they belong to different universality classes.

Chao-Ming Jian is supported by the Gordon and Betty Moore Foundations EPiQS Initiative through Grant GBMF4304. Cenke Xu is supported by NSF Grant No. DMR-1920434, and the David and Lucile Packard Foundation. This work was performed in part at Aspen Center for Physics, which is supported by National Science Foundation grant PHY-1607611.

\footnotetext{
1 X. G. WEN, International Journal of Modern Physics B 04, $239 \quad$ (1990),

https://doi.org/10.1142/S0217979290000139,

URL
} 
https://doi.org/10.1142/S0217979290000139

2 X. Chen, Z.-C. Gu, Z.-X. Liu, and X.-G. Wen, Phys. Rev. B 87, 155114 (2013).

3 X. Chen, Z.-C. Gu, Z.-X. Liu, and X.-G. Wen, Science 338, 1604 (2012)

${ }^{4}$ T. Senthil, A. Vishwanath, L. Balents, S. Sachdev, and M. P. A. Fisher, Science 303, 1490 (2004).

5 T. Senthil, L. Balents, S. Sachdev, A. Vishwanath, and M. P. A. Fisher, Phys. Rev. B 70, 144407 (2004).

${ }^{6}$ A. W. Sandvik, Phys. Rev. Lett. 98, 227202 (2007), URL https://link.aps.org/doi/10.1103/PhysRevLett.98. 227202

7 H. Shao, W. Guo, and A. W. Sandvik, Science 352, 213 (2016), ISSN 0036-8075, https://science.sciencemag.org/content/352/6282/213.full.pd URL https://science.sciencemag.org/content/352/ $6282 / 213$

8 R. G. Melko and R. K. Kaul, Phys. Rev. Lett. 100, 017203 (2008), URL https://link.aps.org/doi/ 10.1103/PhysRevLett.100.017203.

${ }^{9}$ C. Wang, A. Nahum, M. A. Metlitski, C. Xu, and T. Senthil, Phys. Rev. X 7, 031051 (2017), URL https: //link.aps.org/doi/10.1103/PhysRevX.7.031051.

${ }^{10}$ Y. Q. Qin, Y.-Y. He, Y.-Z. You, Z.-Y. Lu, A. Sen, A. W. Sandvik, C. Xu, and Z. Y. Meng, Phys. Rev. X 7, 031052 (2017), URL https://link.aps.org/doi/10. 1103/PhysRevX.7.031052.

11 Z. Bi and T. Senthil, Phys. Rev. X 9, 021034 (2019), URL https://link.aps.org/doi/10.1103/PhysRevX.9. 021034

12 L. Fidkowski and A. Kitaev, Phys. Rev. B 81, 134509 (2010).

13 L. Fidkowski and A. Kitaev, Phys. Rev. B 83, 075103 (2011).

14 L. Fidkowski, X. Chen, and A. Vishwanath, Phys. Rev. X 3, 041016 (2013).

15 C. Wang and T. Senthil, Phys. Rev. B 89, 195124 (2014).

16 Y.-Z. You and C. Xu, Phys. Rev. B 90, 245120 (2014).

17 X.-L. Qi, New J. Phys. 15, 065002 (2013).

18 S. Ryu and S.-C. Zhang, Phys. Rev. B 85, 245132 (2012).

19 H. Yao and S. Ryu, Phys. Rev. B 88, 064507 (2013).

20 Z.-C. Gu and M. Levin, arXiv:1304.4569 (2013).

21 S. Liu, A. Vishwanath, and E. Khalaf, arXiv:1809.01636 (2018).

22 X.-C. Wu, Y. Xu, C.-M. Jian, and C. Xu, arXiv:1906.07191 (2019).

23 Y.-H. Zhang, D. Mao, Y. Cao, P. Jarillo-Herrero, and T. Senthil, Phys. Rev. B 99, 075127 (2019), URL https: //link.aps.org/doi/10.1103/PhysRevB.99.075127.

24 B. L. Chittari, G. Chen, Y. Zhang, F. Wang, and J. Jung, Phys. Rev. Lett. 122, 016401 (2019), URL https://link. aps.org/doi/10.1103/PhysRevLett.122.016401.

20 J. Y. Lee, E. Khalaf, S. Liu, X. Liu, Z. Hao, P. Kim, and A. Vishwanath, arXiv:1903.08130 (2019).

26 J. Liu, Z. Ma, J. Gao, and X. Dai, arXiv:1903.10419 (2019).

27 G. Chen, A. L. Sharpe, E. J. Fox, Y.-H. Zhang, S. Wang, L. Jiang, B. Lyu, H. Li, K. Watanabe, T. Taniguchi, et al., arXiv:1905.06535 (2019).

28 A. L. Sharpe, E. J. Fox, A. W. Barnard, J. Finney, K. Watanabe, T. Taniguchi, M. A. Kastner, and D. Goldhaber-Gordon, arXiv:1901.03520 (2019).

29 N. Bultinck, S. Chatterjee, and M. P. Zaletel, arXiv:1901.08110 (2019).

30 Y.-H. Zhang, D. Mao, and T. Senthil, arXiv:1901.08209
(2019).

31 P. Kim, Ferromagnetic superconductivity in twisted double bilayer graphene, http://online.kitp.ucsb.edu/ online/bands_m19/kim/ (2019), Talks at KITP, Jan 15, 2019.

32 C. Shen, N. Li, S. Wang, Y. Zhao, J. Tang, J. Liu, J. Tian, Y. Chu, K. Watanabe, T. Taniguchi, et al., arXiv:1903.06952 (2019).

33 X. Liu, Z. Hao, E. Khalaf, J. Y. Lee, K. Watanabe, T. Taniguchi, A. Vishwanath, and P. Kim, arXiv:1903.08130 (2019).

${ }^{34}$ Y. Cao, D. Rodan-Legrain, O. Rubies-Bigorda, J. M. Park, K. Watanabe, T. Taniguchi, and P. Jarillo-Herrero, arXiv:1903.08596 (2019).

X. G. Wen and A. Zee, Phys. Rev. B 46, 2290 (1992), URL https://link.aps.org/doi/10.1103/PhysRevB.46.2290

36 X. G. Wen, Phys. Rev. Lett. 64, 2206 (1990), URL https : //link.aps.org/doi/10.1103/PhysRevLett.64.2206

37 X.-G. WEN, International Journal of Modern Physics B 06, 1711 (1992), https://doi.org/10.1142/S0217979292000840, URL https://doi.org/10.1142/S0217979292000840.

38 F. D. M. Haldane, Phys. Rev. Lett. 74, 2090 (1995), URL https://link.aps.org/doi/10.1103/PhysRevLett.74. 2090 .

39 M. Levin, Phys. Rev. X 3, 021009 (2013), URL https: //link.aps.org/doi/10.1103/PhysRevX.3.021009.

40 Z. Bi, R. Zhang, Y.-Z. You, A. Young, L. Balents, C.-X. Liu, and C. Xu, Phys. Rev. Lett. 118, 126801 (2017), URL https://link.aps.org/doi/10. 1103/PhysRevLett.118.126801

${ }^{41}$ Y.-Z. You, Z. Bi, D. Mao, and C. Xu, Phys. Rev. B 93, 125101 (2016), URL http://link.aps.org/doi/10.1103/ PhysRevB.93.125101.

${ }^{42}$ Y.-Z. You, Z. Bi, A. Rasmussen, M. Cheng, and C. Xu, New Journal of Physics 17, 075010 (2015), 1404.6256.

43 H. Song, S.-J. Huang, L. Fu, and M. Hermele, Phys. Rev. X 7, 011020 (2017), URL https://link.aps.org/doi/10. 1103/PhysRevX.7.011020.

${ }^{44}$ Y.-M. Lu and A. Vishwanath, Phys. Rev. B 86, 125119 (2012).

45 H. Song, S.-J. Huang, L. Fu, and M. Hermele, Phys. Rev. X 7, 011020 (2017), URL https ://link .aps .org/doi/10. 1103/PhysRevX.7.011020.

${ }^{46}$ A. Vishwanath and T. Senthil, Phys. Rev. X 3, 011016 (2013), URL https://link.aps.org/doi/10. 1103/PhysRevX.3.011016.

47 C. Xu and T. Senthil, Phys. Rev. B 87, 174412 (2013).

48 Z. Bi, A. Rasmussen, K. Slagle, and C. Xu, Phys. Rev. B 91, 134404 (2015), URL https://link.aps.org/doi/10. 1103/PhysRevB.91.134404

49 C.-M. Jian, Z. Bi, and C. Xu, Phys. Rev. B 97, 054412 (2018), URL https://link.aps.org/doi/10. 1103/PhysRevB.97.054412

${ }^{50}$ F. D. M. Haldane, Phys. Lett. A 93, 464 (1983).

51 F. D. M. Haldane, Phys. Rev. Lett. 50, 1153 (1983).

52 T.-K. Ng, Phys. Rev. B 50, 555 (1994), URL https:// link.aps.org/doi/10.1103/PhysRevB.50.555

53 X. Chen, L. Fidkowski, and A. Vishwanath, Phys. Rev. B 89, 165132 (2014), URL https://link.aps .org/doi/10. 1103/PhysRevB.89.165132

${ }^{4}$ P. Bonderson, C. Nayak, and X.-L. Qi, J. Stat. Mech. p. P09016 (2013).

55 C. Wang, A. C. Potter, and T. Senthil, Phys. Rev. B 
88, 115137 (2013), URL https://link.aps.org/doi/10. 1103/PhysRevB.88.115137

50 M. A. Metlitski, C. L. Kane, and M. P. A. Fisher, Phys. Rev. B 92, 125111 (2015), URL https://link.aps.org/ doi/10.1103/PhysRevB.92.125111.

5r H. Lieb, T. D. Schultz, and D. C. Mattis, Ann. Phys. 16, 407 (1961).
58 M. Oshikawa, Phys. Rev. Lett. 84, 1535 (2000), URL https://link.aps.org/doi/10.1103/PhysRevLett.84. 1535 .

59 M. B. Hastings, Phys. Rev. B 69, 104431 (2004), URL http://link.aps.org/doi/10.1103/PhysRevB.69. 104431 